\title{
Letter to the editor: The dynamic casein supramolecule: A response to Horne (2010)
}

\author{
D. J. McMahon ${ }^{1}$ \\ Western Dairy Center, Utah State University, Logan 84322-8700
}

I agree with Horne (2010) that there should be an open discussion of the terminology used for describing what have traditionally been called casein micelles and which I have referred to in McMahon and Oommen (2008) and McMahon et al. (2009) as casein supramolecules. And rather than being an arbitrary change by the Journal of Dairy Science, use of the term "casein supramolecule" was the choice of the authors and came about because the treatment of milk by cooling, heating, acidifying, and calcium-chelating results in colloidal particles with structures that are different from casein micelles as they are secreted from the mammary gland. This is a similar problem to defining the native structure of the casein molecules themselves, in that their environment dictates their tertiary structure.

A surfactant micelle is a good example to use for teaching about self-assembled supramolecules in which the molecular entities are held together and organized by means of noncovalent intermolecular interactions. When teaching students who are just beginning a study of milk chemistry, it is usually necessary to explain that "casein micelles" are not analogous to such surfactant micelles. This is not an issue for senior dairy scientists who are already well versed in dairy chemistry and have used the term casein micelles for many years.

Received May 6, 2010.

Accepted May 26, 2010.

${ }^{1}$ Corresponding author: djm@cc.usu.edu
The mechanism for transporting calcium phosphate from the mammary glands is indeed a marvel of nature. Keeping the name "casein micelle" would honor those scientists who pioneered its study. However, recognizing it as a dynamic supramolecule whose structural elements respond to environment changes would provide a perspective that further portrays its uniqueness.

When describing phosphate contained within the casein supramolecule it is common to divide it into 2 classes: organic and inorganic phosphate (e.g., Françoise et al., 2009). Organic phosphate refers to phosphate that is covalently bound to the caseins as part of the phosphoserine side chains. In contrast, inorganic phosphate is present as calcium phosphate nanoclusters that can be released from the supramolecule upon acidification. Perhaps there is some sharing of electrons between the phosphoserines and the calcium phosphate nanoclusters, but I would classify their interaction as ionic rather than covalent, thus meeting the definition of noncovalent interactions between molecular entities as defined for supramolecules.

\section{REFERENCES}

Françoise, K. A., T. Kablan, A. Kamenan, and A. Lagaude. 2009. Rheological and biochemical properties of acidified milk/pectin cogels. Eur. J. Sci. Res. 25:584-596.

Horne, D. S. 2010. Casein micelles or casein supramolecules? J. Dairy Sci. 93:3403.

McMahon, D. J., H. Du, W. R. McManus, and K. M. Larsen. 2009. Microstructural changes in casein supramolecules during acidification of skim milk. J. Dairy Sci. 92:5854-5867. doi:10.3168/ jds.2009-2324.

McMahon, D. J., and B. S. Oommen. 2008. Supramolecular structure of the casein micelle. J. Dairy Sci. 91:1709-1721. 